\author{
Hindsight Bias in Depression \\ Julia Groß \\ Heinrich-Heine-Universität Düsseldorf \\ Hartmut Blank \\ University of Portsmouth \\ Ute J. Bayen \\ Heinrich-Heine-Universität Düsseldorf
}

Author Note

Julia Groß, Institute for Experimental Psychology, Heinrich-Heine-Universität Düsseldorf, Düsseldorf, Germany; Hartmut Blank, Department of Psychology, University of Portsmouth, Portsmouth, U.K.; Ute Johanna Bayen, Institute for Experimental Psychology, Heinrich-Heine-Universität Düsseldorf, Düsseldorf, Germany.

We thank Siegmund Switala, Albert-Georg Lang, and Lennart Friese for technical assistance, and Anna-Christina Breuer, Doris Dahlke, and Laura Richter for help with data collection.

Correspondence concerning this article should be addressed to Julia Groß, HeinrichHeine-Universität Düsseldorf, Institute for Experimental Psychology, Building 23.02., Universitätsstr. 1, D-40225 Germany, E-Mail: gross@hhu.de. 


\begin{abstract}
People tend to be biased by outcome knowledge when looking back on events. This phenomenon is known as hindsight bias. Clinical intuition and theoretical accounts of affectregulatory functions of hindsight bias suggest a link between hindsight bias and depression, but empirical evidence is scarce. In two experiments, participants with varying levels of depressive symptoms imagined themselves in everyday scenarios that ended positively or negatively, and completed hindsight and affect measures. Participants with higher levels of depression judged negative outcomes, but not positive outcomes, as more foreseeable and more inevitable in hindsight. They also recalled prior expectations about outcomes as more negative for negative, but not for positive outcomes. This memory hindsight bias was accompanied by disappointment, suggesting a relation to affect-regulatory malfunction. We propose that "depressive hindsight bias" indicates a negative schema of the past, and that it sustains negative biases in depression.
\end{abstract}

Keywords: hindsight bias, judgment bias, memory bias, individual differences, depression 


\section{Hindsight Bias in Depression}

Knowing how an event turned out makes it difficult to reconstruct one's prior prognosis. Specifically, we overestimate in hindsight what we predicted in foresight regarding outcomes of, for example, football matches (Roese \& Maniar, 1997), elections (Blank, Fischer, \& Erdfelder, 2003), medical assessments (Arkes, 2013), scientific studies (Slovic \& Fischhoff, 1977), or romantic dates (Carli, 1999). This phenomenon is known as hindsight bias (for a review, see Roese \& Vohs, 2012). The cognitive processes involved in hindsight bias are well understood, but little is known about its role in affective selfregulation and depression. Did I see the accident coming? Did I know all along that my application would not be successful? Was my marriage likely to be happy, or were the chances not too good to begin with? How we look back on our personal past may be biased by how we experience and judge the present. In turn, the way we look back on our past may influence how we presently feel and will thus have consequences for emotional well-being. Cognitive biases are a core symptom of depression and result from the activation of negative schemata (e.g., Beck, Rush, Emery, \& Shaw, 1979). In the research reported in this paper, we investigated how individual differences in hindsight bias are related to depressive symptoms. We show that hindsight bias is a core characteristic of depressive cognition, and we will argue that it may constitute a mechanism that corroborates negative schemata. Before introducing our studies, we review different manifestations of hindsight bias and discuss their role in affect and depression.

\section{Hindsight Bias}

Hindsight bias encompasses three dissociable manifestations: foreseeability impressions, inevitability impressions, and memory hindsight bias (Blank, Nestler, von Collani, \& Fischer, 2008; Roese \& Vohs, 2012). Foreseeability impressions occur when we think that we knew all along how events would turn out, or that we could have predicted 
certain outcomes, for example, stock developments (Louie, 1999) or a nuclear power plant accident (Oeberst, von der Beck, \& Nestler, 2014). Foreseeability impressions refer to our own past mental states regarding the outcome ("I knew it all along"). Consequently, these judgments strongly depend on metacognitive input, such as the level of surprise (Calvillo \& Gomes, 2011).

Inevitability impressions are biased representations of the objective state or functioning of the world, after having received outcome knowledge ("It had to happen"). Outcomes seem more probable, necessary, or inevitable in hindsight (e.g., outcomes of business decisions, political strategies, and athletic competitions). A plausible causal connection of events leading to an outcome increases coherence and flow, and consequently its perceived inevitability. Or, as Roese and Vohs (2012) put it: "[...] the better the story, the more hindsight bias" (p. 4). Although foreseeability and inevitability impressions are often aligned, they can be empirically dissociated (e.g., when the true causes of event outcomes become known only after the fact, which mitigates foreseeability impressions but boosts inevitability impressions; Blank \& Nestler, 2006; Nestler, Blank, \& Egloff, 2010).

Memory hindsight bias occurs when recollections of earlier judgments are distorted by outcome knowledge. For example, a person may estimate the Berlin TV tower to be 310 meters tall, receive factual feedback (368 meters), and in a later test reconstruct the initial estimate closer to the correct solution (e.g., 320 meters; Erdfelder \& Buchner, 1998; Hawkins \& Hastie, 1990). This may happen because the knowledge that forms the basis for the reconstructed judgment has been updated with the factual feedback (e.g., Hoffrage, Hertwig, \& Gigerenzer, 2000, and Pohl, Eisenhauer, \& Hardt, 2003). In this sense, memory hindsight bias constitutes a by-product of knowledge updating (Hoffrage et al., 2000). Aside from cognitive explanations, motivational factors have been suggested to underlie biased memory 
reconstruction (e.g., the motive to appear knowledgeable); however, empirical evidence is so far weak (Hawkins \& Hastie, 1990; Musch \& Wagner, 2007).

\section{Hindsight Bias and Affect Regulation}

Whether we perceive a personally relevant and negative outcome (e.g., a relationship break-up or a failed exam) as having been foreseeable or surprising may influence how we feel about the event and about ourselves. Yet, in its long research history, hindsight bias has been primarily conceived as a cognitive phenomenon. Only few articles were concerned with a potential impact of hindsight bias on affective states, or with an affect-regulatory function of hindsight bias. These studies investigated outcomes that are personally relevant and negative, for example, a layoff (Mark \& Mellor, 1991), a failed stock purchase (Mark, Boburka, Eyssell, Cohen, \& Mellor, 2003), high cholesterol levels (Renner, 2003), exam grades (Haslam \& Jayasinghe, 1995), a defeat of one's favorite soccer team (Tykocinski, Pick, \& Kedmi, 2002), or missing out on a sale (Blank \& Peters, 2010). According to these studies, the distortion of outcome probabilities in retrospect (i.e., hindsight bias) is related to the occurrence, intensity, and duration of affective states in several ways.

Foreseeability and regret. One way to deal with negative outcomes is to deny their foreseeability. If one did not see the outcome coming, one cannot be held responsible. Thus, the foreseeability of an outcome is a necessary precursor of regret, (self-)blame, and legal liability (see Alicke, 2000, for a theoretical contribution on blame). The strategy to deny an outcome's foreseeability has been labeled defensive processing and is supposedly selfprotective in high-control situations (i.e., when personal decisions play a role). Defensive processing was found in several studies (Blank \& Peters, 2010; Louie, 1999, 2005; Louie, Curren, \& Harich, 2000; Mark et al., 2003; Mark \& Mellor, 1991; Pezzo \& Beckstead, 2008). Blank and Peters (2010) found larger foreseeability of negative outcomes to be associated 
with higher levels of self-blame, supporting the notion of an affect-regulatory function of a denial of foreseeability.

Inevitability and disappointment. Viewing an event as inevitable in retrospect may reduce disappointment: If something negative was bound to happen, there was no way to avoid it. This strategy has been labeled retroactive pessimism (Tykocinski, 2001), and its regulatory function is presumably conditional on low-control situations (i.e., where situational factors play a role; Tykocinski \& Steinberg, 2005). Retroactive pessimism was reported in several studies (Blank \& Peters, 2010; Tykocinski, 2001; Tykocinski et al., 2002; Tykocinski \& Steinberg, 2005; Wann, Grieve, Waddill, \& Martin, 2008). Yet, whereas Blank and Peters (2010) found a negative correlation between hindsight bias and disappointment (interpreted as a role of hindsight bias in reducing disappointment), Wann and colleagues (2008) found a positive correlation (interpreted as a higher need for coping via hindsight bias when disappointment is high). Tykocinski and Steinberg (2005, Exp. 2) found disappointment to be unaffected by hindsight bias, but pointed to the possibility that hindsight bias may have already been effective when disappointment was assessed. Thus, the exact nature of the link between hindsight bias and negative affect is still unclear.

\section{The Role of Hindsight Bias in Depression}

Depressive disorders are among the most common mental disorders and place an enormous burden on individuals and society (e.g., Üstün, Ayuso-Mateos, Chatterji, Mathers, \& Murray, 2014; Wittchen \& Jacobi, 2005). Impairment of the regulation of negative mood and emotion plays a significant role in the onset and maintenance of depression (Gotlib \& Joormann, 2010), and cognitive processes figure prominently in this regulation (see Beck \& Bredemeier, 2016, for an integrative model of depression). According to Beck's theory of depression (Beck et al., 1979), negative schemata result in negative biases in attention, memory, and thinking which in turn contribute to depressive symptoms, specifically negative 
affect. Generally, schemata are organized knowledge or belief structures about aspects of the world. Beck's theory builds on decades of research by cognitive psychologists who demonstrated the biasing influences of schemata on memory, judgment, and other cognitive functions (e.g., Alba \& Hasher, 1983; Bartlett, 1932; Bayen, Nakamura, Dupuis, \& Yang, 2000; Brewer, 2000; Cantor \& Mischel, 1977; Kuhlmann, Vaterrodt, \& Bayen, 2012).

With the present research, we seek to investigate if hindsight bias operates differently in individuals with depressive symptoms, and if it is related to affective responding. To experience disappointment and regret from time to time is adaptive, because these emotions provide valuable feedback that leads us to adjust our expectations or make better decisions in the future (Zeelenberg, van Dijk, \& Manstead, 1998). Yet, individuals with depression struggle with the regulation of negative affect when it exceeds its informative function. Foreseeability decreases or inevitability increases for negative events are forms of reappraisal, and thus have the potential to regulate negative affect (i.e., they can be adaptive manifestations of hindsight bias). However, reappraisal may be difficult for individuals who suffer from depressive symptoms, for example, during a depressive episode or a traumarelated disorder. Trauma survivors often believe that unexpected traumatic events were foreseeable - a mistaken belief that can contribute to intense feelings of regret and guilt (Kubany \& Manke, 1995), particularly when personal responsibility is assumed for the event. Clinical practitioners hence frequently notice maladaptive manifestations of hindsight bias in clinical contexts ("the phenomenon is commonplace", Kubany \& Manke, 1995, p. 30; Leahy, 2004), but note that hindsight bias "has been studied primarily in social psychology research [...] and the term is not used widely in the clinical literature" (Kubany \& Manke, 1995, p. 29).

Hence, there is little empirical research on the role of hindsight bias in depression. Haslam and Jayasinghe (1995) found memory hindsight bias for predicted exam grades more 
frequently in students with elevated (but sub-clinical) depression scores, and this effect was present for both optimistic and pessimistic grade predictions. Groß and Bayen (2016) found higher memory hindsight bias for numerical judgments in participants with elevated depression scores. Other evidence for increased hindsight bias in depression is mostly anecdotal (e.g., Williams, 1992). It is plausible that hindsight bias fuels negative affect in a similar fashion as the cognitive errors specified in the cognitive model of depression (Beck et al., 1979). Yet, to our knowledge, the two cited empirical studies are the only ones to investigate if hindsight bias occurs more frequently or more intensely with higher levels of depression. Furthermore, it is unknown whether a potential depressive hindsight perspective manifests itself in foreseeability, inevitability, memory bias, or all of these.

The goal of Experiment 1 was to fill this gap by investigating two main questions. First, are judgments of foreseeability and inevitability of past positive and negative event outcomes related to level of depression? Second, are these hindsight judgments related to positive or negative affect following the outcomes? The goal of Experiment 2 was to replicate and extend the findings of Experiment 1 by investigating three additional questions. First, are pre-to-post outcome shifts in judgments of foreseeability and inevitability (i.e., hindsight bias) related to level of depression? Second, is memory hindsight bias related to level of depression? Third, is hindsight bias related to other forms of depressive cognition (i.e., negative schemata, dysfunctional attitudes, and cognitive errors; Beck et al., 1979)?

In addition, we expected to replicate established findings regarding the relationship between depression and two characteristic cognitive patterns: First, taking responsibility for negative, but not positive outcomes (a typical attributional style in depression; Sweeney, Anderson, \& Bailey, 1986); and second, a tendency to expect negative outcomes (Beck et al., 1979). 
In both experiments, we adopted a dimensional view of depression on conceptual and measurement grounds. Conceptually, there is growing evidence for a latent dimensional (rather than taxonic) structure of depression, as found via taxometric analyses of depressive symptoms in both clinical and general population-based samples (e.g., Hankin, Fraley, Lahey, \& Waldman, 2005; Ruscio \& Ruscio, 2000). In addition, measuring depression on a continuum provides greater statistical power for data analyses (e.g., MacCallum, Zhang, Preacher, \& Rucker, 2002). For these reasons, we used nonclinical samples that were recruited to cover a wide distribution of symptom severity from none to high.

\section{Experiment 1}

In Experiment 1, participants with varying levels of depressive symptoms listened to everyday-life scenarios that either had a positive or a negative outcome. They imagined themselves in these situations and subsequently judged the outcomes' foreseeability and inevitability, their own responsibility for the outcomes, and their affective reactions to the outcomes (regret, disappointment, joy, and pride).

Prior research suggests that healthy and depressed individuals differ primarily in their processing of negative information (Gotlib \& Joormann, 2010). Therefore, with positive outcomes, we expected no relationship between depressive symptoms and impressions of inevitability and foreseeability.

We expected participants with higher levels of depression to evaluate negative outcomes as more foreseeable. If denial of foreseeability (i.e., defensive processing) is an effective self-regulation strategy, then it should less likely occur with higher levels of depression.

Predictions regarding inevitability impressions seemed less clear-cut, and we considered two possibilities. On the one hand, higher levels of depression may be associated with lower perceived inevitability (i.e., less retroactive pessimism). If retroactive pessimism 
is an adaptive way of affect regulation, individuals with depressive symptoms may not be able to use it as adaptively as individuals without depressive symptoms. On the other hand, higher levels of depression may be associated with higher perceived inevitability because of depressed individuals' tendency to perceive themselves as helpless and as victims of fate (Abramson, Seligman, \& Teasdale, 1978; Alloy \& Abramson, 1982; Andersen, 1990).

We had several hypotheses regarding affect. As negative affect is a core symptom of depression, we expected negative affect to be stronger with higher levels of depression. Additionally, we expected negative affect after negative outcomes to be stronger with higher perceived responsibility. This should hold specifically for the emotion of regret, due to its strong relationship to self-agency and thus responsibility (e.g., Zeelenberg et al., 1998). Regarding affective consequences of hindsight judgments, regret should be stronger with higher ratings of foreseeability, if decreasing an outcome's foreseeability in retrospect is associated with a reduction of regret (defensive processing). If increasing an outcome's inevitability in retrospect is associated with a reduction of disappointment (retroactive pessimism), disappointment should be lower with higher ratings of inevitability.

\section{Method}

Participants. We preselected participants with regard to depressive symptoms. To this end, we screened 245 students at Heinrich-Heine-Universität Düsseldorf using the German version of the Beck Depression Inventory (BDI-II), a self-report measure of depressive symptoms in normal populations (Hautzinger, Keller, \& Kühner, 2006). We invited respondents for participation based on their agreement to be contacted, and based on their BDI-II score. A BDI-II score of > 13 corresponds to at least mild depressive symptoms according to Beck, Steer, and Brown (1996). We recruited a similar number of participants from both above and below this cut-off, approximately matched according to age and gender. This recruitment procedure allowed us to obtain a sample with sufficient variation on the 
depression continuum. Participant characteristics are shown in Table 1. All 60 participants of the laboratory experiment were either native speakers of German or had been living in Germany since the age of 6 or earlier. They received either course credit or $€ 8$.

Design. Outcome valence (positive vs. negative) of the scenarios was a categorical variable and manipulated within participants. Depressive symptoms (i.e., BDI-II score), hindsight cognitions (inevitability, foreseeability), responsibility, and affective reactions were continuous measured variables.

Materials and measures. All materials and measures were in German. We created 16 scenario descriptions ranging in length from 47 to 147 words. Each scenario described a situation in a domain of everyday life (work, performance, family, leisure, social, romantic) applicable to different age groups and educational backgrounds. For each scenario, we created a version with a positive outcome and a version with a negative outcome. The following is an example of a scenario description, followed by its positive outcome and its negative outcome:

Imagine that after the past stressful weeks you have now finally arrived at your vacation destination in Southern France. Via the internet, you have booked a room in a small hotel at the seaside.

Arriving at the hotel, you realize that the room looks even better than what you saw on the internet. You have a balcony with ocean view, and the staff has equipped your room with a basket full of fresh fruit. (positive outcome) 
Arriving at the hotel, you realize that the room does not look at all like what you saw on the internet. It is small, shabby, and lacks the ocean view that you had expected. (negative outcome)

According to a pilot study with 19 raters, none of these outcomes was perceived as either absolutely uncontrollable or absolutely controllable. That is, each scenario received a mean rating $>2$ or $<6$ on a 7 -point scale ranging from 1 ("not at all controllable") to 7 (“absolutely controllable”).

We assigned the 16 scenarios to two sets of eight scenarios each (Sets 1 and 2), with a comparable mixture of topics. We then created two versions of the materials: In Version 1, Set 1 had positive and Set 2 had negative outcomes; in Version 2, Set 1 had negative and Set 2 had positive outcomes. Thirty participants received Version 1, and 30 received Version 2, with similar BDI-II score distributions for Version $1(M=11.1, S D=9.4)$ and Version $2(M$ $=12.2, S D=8.4)$.

We measured foreseeability and inevitability with a set of established items (Blank et al., 2008; Blank \& Peters, 2010), minimally adapted to the purposes of the present research. Three items measured foreseeability ("I knew all along how the situation would turn out", "It was difficult to predict how the situation would turn out" [ reverse coded], "I had a clear vision of how the situation would turn out"). Two items measured inevitability ("Under the given circumstances the outcome of the situation was essentially determined", "Because of the many factors that could influence the outcome of the situation, the outcome was still open" [reverse coded]). Further, we created one imaginability item ("I can clearly imagine myself in the situation"), one responsibility item ("If I was in the described situation, I'd feel responsible for the described outcome"), as well as four affect items measuring two positive and two negative affective reactions (joy, pride, regret, disappointment). Each affect item 
started with "If I was in the described situation, I'd feel...". Participants marked their degree of approval for each item by adjusting an arrow on a continuous scale ranging from "I fully disagree" to "I fully agree." We translated all of these ratings into scores between 0 and 100.

Procedure. Participants were tested individually or in groups of up to five. They were seated in individual computer booths, gave informed consent, and completed the BDI-II (we used the resulting BDI-II score for all analyses). They then listened to the 16 scenarios via headphones, in an order randomized by participant. Following each outcome, participants rated the imaginability of the scenario, hindsight cognitions, responsibility, and affect (in this order). Finally, participants filled out a demographics and health questionnaire and were debriefed and compensated.

\section{Data Analyses and Results}

General approach to data analysis: Multilevel modeling. Multilevel modeling (MLM) is the method of choice for our data analyses for two reasons. First, this approach allowed us to analyze interactions between hierarchically crossed levels of data (e.g., between hindsight cognitions measured at the scenario level and BDI-II scores measured at the participant level) without losing information and power due to data aggregation. Second, we were interested in the interaction between a categorical design factor (outcome valence) and a continuous person-level variable (BDI-II score). This precludes analyses within a traditional ANCOVA approach, which requires homogeneous regression slopes (for an introduction to MLM of experimental data, see, e.g., Hoffman \& Rovine, 2007).

Multilevel modeling allowed us to estimate overall effects of our predictor variables (i.e., fixed effects) while accounting for several sources of variation that are not of theoretical interest, because they reflect idiosyncracies of participants or scenarios (i.e., random effects). Specifically, we accounted for variability between individual participants and scenarios 
(random intercepts), and for variability in predictor effects between participants and between scenarios (random slopes). ${ }^{1}$

Fixed-effect predictors at the scenario level (e.g., responsibility as a predictor of affect) were centered at the scenario level, that is, the scenario mean was subtracted from each individual predictor score. This group mean centering is recommended if interactions between the data levels are of interest, and to avoid problems due to multicollinearity (Enders \& Tofighi, 2007). We did not center BDI-II score as a fixed-effect predictor on the person (highest) level.

We present the regression weight estimates $b$ for the respective fixed effects, along with their respective $p$ values. ${ }^{2}$ Alpha was set to .05 for all statistical tests reported in this article.

\section{Preliminary analyses.}

Imaginability of scenarios. We considered it important that participants be able to imagine themselves in the scenarios. This was the case; mean imaginability of the scenarios was satisfactory with $M=80.87(S D=13.41)$ on the $0-100$ scale. In addition, imaginability of the scenarios was neither affected by BDI-II score $(b=-.19, p=.378)$, nor by scenario outcome valence $(b=1.23, p=.583)$, nor by the interaction of the two predictors $(b=-.01, p$ $=.973)$.

Responsibility as a function of BDI-II score and outcome valence. We analyzed responsibility as a function of BDI-II score and outcome valence to replicate the well-known responsibility attribution pattern (Sweeney et al., 1986). We found a main effect of BDI-II score, $b=0.83, p<.001$, indicating higher responsibility ratings with higher BDI-II scores. We also found a main effect of outcome valence, $b=14.44, p=.003$, with higher responsibility ratings for positive compared to negative outcomes. Importantly, there was an interaction of the two predictors, $b=-1.27, p<.001$. Decomposition of the interaction 
revealed that for negative outcomes, responsibility ratings were higher with higher BDI-II scores, $b=0.83, p<.001$, but for positive outcomes, responsibility ratings were lower with higher BDI-II scores, $b=-0.45, p=.046$. Thus, as level of depression increased, higher responsibility was taken for negative outcomes, but less responsibility was taken for positive outcomes, confirming the established attributional pattern (Sweeney et al., 1986).

Main analyses: Overview. To answer our two main research questions, we (1) analyzed hindsight cognitions (foreseeability, inevitability) as a function of BDI-II score and outcome valence (positive vs. negative). We (2) analyzed affect ratings (regret and disappointment after negative outcomes; pride and joy after positive outcomes) as a function of hindsight cognitions, responsibility, and BDI-II score. Descriptive statistics are displayed in Table 2, separately for positive and negative scenario outcomes, and - for illustration purposes - separately for participants with no or minimal $(\leq 13)$ and elevated $(>13)$ BDI-II scores.

\section{Hindsight cognitions as a function of BDI-II score and outcome valence. We} averaged the three foreseeability items and the two inevitability items into composite scores for each participant and each scenario (mean Cronbach's $\alpha=.85$ and .82 , respectively). Results are shown in the upper panels of Figure 1 (with foreseeability and inevitability averaged across scenarios per participant for ease of presentation). We found a main effect of BDI-II score, $b=0.74, p=.001$, indicating higher foreseeability ratings with higher BDI-II scores. Importantly, there was an interaction of BDI-II score and outcome valence, $b=-0.68$, $p=.005$. Decomposition of the interaction revealed that foreseeability ratings were higher with higher BDI-II scores for negative outcomes only, $b=0.74, p=.001$, not for positive outcomes, $b=0.06, p=.755$.

The same pattern emerged for inevitability ratings: We found a main effect of BDI-II score, $b=0.79, p<.001$, and an interaction of BDI-II score and valence, $b=-0.72, p=.004$. 
Inevitability ratings were higher with higher BDI-II scores for negative, $b=0.79, p<.001$, but not for positive outcomes, $b=0.07, p=.706$.

Affect as a function of hindsight cognitions, responsibility, and BDI. The previous analyses showed that for negative outcomes, elevated levels of depressive symptoms went along with elevated hindsight judgments. The next set of analyses explored how these hindsight judgments related to affective reactions. In addition to hindsight judgments (foreseeability, inevitability), we included responsibility and BDI-II score as predictors of negative and positive affect.

Negative affect (after negative outcomes). As foreseeability decreases have been associated with decreases in regret, we analyzed regret in response to the negative scenario outcomes as a function of foreseeability, responsibility, and BDI-II score. We found no main effects, but an interaction of foreseeability, responsibility, and BDI-II score, $b=0.0004, p=$ .015. To decompose this interaction, we performed separate analyses for participants with elevated (i.e., mild to severe) depressive symptoms (BDI-II >13) versus those with no or low symptoms (BDI-II $\leq 13)$. For the latter, we found no significant effects. For those with elevated symptoms, we found a main effect of responsibility, $b=0.226, p=.019$, and an interaction of responsibility and foreseeability, $b=0.005, p=.017$. Thus, participants with elevated symptoms imagined greater regret after negative outcomes when they assumed greater responsibility for these outcomes in combination with higher foreseeability of these outcomes.

As inevitability increases have been associated with decreases in disappointment, we analyzed disappointment in response to the negative scenario outcomes as a function of inevitability, responsibility, and BDI-II. We found no main effects or interactions.

Positive affect (after positive outcomes). We had no clear predictions regarding effects of hindsight cognitions on positive affect. Nonetheless, we analyzed affective 
reactions in parallel to the above analyses: We treated pride as the positive counterpart of regret, as both of these emotions are related to thoughts about personal choice and behavior, and we treated joy as the positive counterpart of disappointment, as both of these emotions are related to thoughts about situational factors (Zeelenberg, Van Dijk, Manstead, \& van der Pligt, 2000).

An analysis of pride after positive scenario outcomes as a function of foreseeability, responsibility, and BDI-II yielded a main effect of BDI-II score only, $b=-0.36, p=.033$, with participants with lower BDI-II scores experiencing more pride. A corresponding analysis of joy after positive outcomes as a function of inevitability, responsibility, and BDIII score again showed the same main effect of BDI-II score only, $b=-0.66, p<.001$, with participants with lower BDI-II scores experiencing more joy.

\section{Discussion}

Two questions were of main interest in Experiment 1. First, do hindsight cognitions following positive and negative outcomes differ as a function of level of depression? The answer is a clear yes. The depressive hindsight perspective is characterized by viewing negative outcomes - but not positive outcomes - as both more foreseeable and more inevitable. Higher foreseeability of negative outcomes in hindsight (i.e., less defensive processing) may reflect the reconstruction of a past pessimistic view of the future (i.e., a negative outcome expectation). Higher inevitability of negative outcomes in hindsight (i.e., retroactive pessimism) may reflect a typical external control fallacy (helplessness) in depression. In this sense, depressive symptoms are not only associated with a negative view of the world, the self, and the future (cognitive triad; Beck et al., 1979), but also with a negative view of the past.

Second, are affective reactions to outcomes associated with these hindsight cognitions (i.e., an association of regret with defensive processing, or disappointment with retroactive 
pessimism)? We found no (clear) indication of this association. However, in accordance with the nature of regret, we found higher ratings of responsibility to be associated with greater regret; and this was specifically the case for individuals with elevated symptoms who rated the negative outcomes as more foreseeable. Also in accordance with prior results (Sweeney et al., 1986), higher levels of depression were associated with a higher sense of responsibility for negative outcomes and a lower sense of responsibility for positive outcomes.

Hindsight, responsibility, and affect thus presumably influence each other in a complex interplay. We examined these influences further in Experiment 2.

\section{Experiment 2}

Experiment 2 was aimed at replicating the finding of elevated hindsight judgments in participants with elevated depressive symptoms. In addition, we sought to determine whether these elevated judgments of foreseeability and inevitability truly reflect hindsight bias, that is, a bias in judgment and/or recall of a prior judgment that occurs due to outcome knowledge. While this is certainly a possibility, higher foreseeability and inevitability of negative outcomes may, alternatively, simply reflect the (unbiased) reconstruction of the foreseeability and inevitability impressions that participants already had before the receipt of outcome knowledge. To be able to decide between these possibilities, we extended the design of Experiment 1 such that participants were asked to rate the foreseeability and inevitability of each scenario both before and after receiving outcome knowledge. This allowed us to compute hindsight-bias scores, that is, difference scores of participants' hindsight (with outcome knowledge) and foresight (without outcome knowledge).

Additionally, we were interested in whether individuals with elevated levels of depression were also more prone to memory hindsight bias (cf. Groß \& Bayen, 2016). Therefore, we asked participants to initially rate their expectation regarding the outcome of each scenario. We expected to find negative outcome expectations that are typical in 
depression (e.g., Beck et al., 1979). After providing outcome feedback, we then asked participants to recall their prior expectation. This allowed us to compute a difference score to measure memory hindsight bias. Moreover, to be able to relate foreseeability, inevitability, and memory hindsight bias to known cognitive dysfunction in depression, we included three established measures of depressive cognition: the Cognitive Triad Inventory (CTI; Beckham, Leber, Watkins, Boyer, \& Cook, 1986), the Dysfunctional Attitudes Scale (DAS; Power et al., 1994), and the Cognitive Errors Questionnaire (CEQ; Lefebvre, 1981). In addition, we included three items to measure participants' current mood state.

\section{Method}

Participants. We screened a new sample of 346 students at the same university with the BDI-II. Entrance criteria and compensation were the same as for Experiment 1. We excluded the data of one participant who was known for cheating in experiments. Table 1 shows the participant characteristics of the 75 remaining participants.

Materials and measures. We used the same 16 scenarios as in Experiment 1. Thirtysix participants received Version 1, and 39 participants received Version 2, again with similar BDI-II score distributions for Version $1(M=13.8, S D=11.2)$ and Version $2(M=11.3, S D$ $=9.3)$.

Ratings were again assessed with the same 0-100 scale. To measure participants' mood, we added the following questions: "At the moment, my mood is [very bad - very good]", "At the moment, my mood is [depressed - cheerful]", and "At the moment, I feel [sad - happy]. We added one item to our existing measure of inevitability impressions ("The outcome of the situation is inevitable"), making it a three-item measure just like the foreseeability measure. Each foreseeability and inevitability item was worded in the present tense (pre-outcome), and past tense (post-outcome, e.g., "The outcome of the situation was inevitable"). To measure outcome expectation (i.e., pre-outcome), participants rated "The 
situation will turn out ..." from 0 (labeled "negative") to 100 (labeled "positive"). To measure recall of the prior expectation rating (post-outcome), participants rated "I assumed that the situation would turn out ..." on the same scale. We used the same responsibility and affect items as in Experiment 1.

We included the German versions of the three measures of depressive cognition: the CTI (Pössel, 2009), a short version of the DAS (DAS-18A; Rojas, Geissner, \& Hautzinger, 2015), and the CEQ (Pössel, 2008). The CTI measures negative schemata of the world, the self, and the future (e.g., "The world is a very hostile place"). The DAS measures negative conditional beliefs that are embedded in these schemata. In individuals with depressive symptoms, these beliefs and attitudes about achievement and failure, dependency and approval, as well as self-control, tend to be rigid and extreme (e.g., "If I fail at my work, I am a failure as a person”). Finally, the CEQ measures negative biases in logic and thinking, such as selective attention to negative information or taking personal responsibility for negative outcomes. These errors result from biased information processing which in turn is triggered by the activation of negative schemata. The questionnaire comprises short vignettes along with a conclusive statement that represents a cognitive error (e.g., personalization: "You just finished spending three hours cleaning the basement. Your spouse, however, doesn't say anything about it. You think to yourself, 'S(he) must think I did a poor job"').

Design. Outcome valence (positive vs. negative) and time of measurement (preoutcome vs. post-outcome) were categorical within-subject variables. BDI-II score, mood, hindsight cognitions (inevitability, foreseeability), responsibility, affective reactions, expectation ratings, and cognitive dysfunction were continuous measured variables.

Procedure. First, participants rated their current mood. Thereafter, the procedure was identical to Experiment 1, except that participants first listened to all of the 16 scenarios without outcome information, and later - in the same randomized order - listened to them 
again along with the outcome. For each scenario, participants were asked to provide preoutcome ratings (imaginability, foreseeability, inevitability, outcome expectation) and postoutcome ratings (foreseeability, inevitability, recalled outcome expectation, responsibility, and affective reactions). After completion of the computer task, participants filled out the DAS-18A, CTI, and CEQ.

\section{Results}

Table 2 shows descriptive statistics for foreseeability, inevitability (pre-outcome and post-outcome), responsibility, affect, expectations (pre-outcome), and recalled expectations (post-outcome).

\section{Preliminary analyses.}

Imaginability of scenarios. Mean imaginability of the scenarios was $M=73.43(S D=$ 15.13), which was descriptively lower than in Experiment 1. We nevertheless consider it satisfactory because ratings were assessed before disclosure of the scenario outcome (i.e., for shorter and less detailed scenario descriptions). In addition, imaginability of the scenarios was affected by BDI-II score, $b=-.38, p=.024$; that is, contrary to Experiment 1 , participants with higher levels of depression were less able to imagine themselves in the scenarios. $^{3}$

Depression and outcome expectations. Participants with higher BDI-II scores expected more negative outcomes, $b=-0.90, p<.001$. This result reflects the negative view of the future that is typical of depression.

Hindsight cognitions as a function of BDI-II score and valence. To replicate the analyses of Experiment 1, we analyzed hindsight cognitions (foreseeability, inevitability) as a function of BDI-II score and outcome valence. To this end, we averaged the three postoutcome foreseeability items and the three post-outcome inevitability items to composite 
scores for each participant and each scenario (mean Cronbach's $\alpha=.83$ and .75 , respectively).

For foreseeability, there were main effects of BDI-II score, $b=0.54, p=.001$, and valence, $b=15.57, p<.001$, that, like in Experiment 1 , were qualified by an interaction, $b=-$ $0.87, p<.001$ : Ratings were higher with higher BDI-II scores for negative outcomes only, $b=$ $0.54, p=.001$, not for positive outcomes, $b=-0.34, p=.085$.

For inevitability, there were main effects of BDI-II score, $b=0.89, p<.001$, and valence, $b=7.75, p=.020$. Again, these effects were qualified by an interaction, $b=-0.49, p$ $=.002$. Different from Experiment 1, however, the positive linear relationship emerged for both types of outcomes, but the relationship for positive outcomes was weaker, $b=0.90, p<$ .001 (negative) versus $b=0.39, p=.022$ (positive).

Thus, we found essentially the same depressive hindsight effect as in Experiment 1: Participants with elevated depressive symptoms showed elevated foreseeability and inevitability impressions after negative outcomes. The aim of the following analyses was to determine whether this result reflects already heightened levels in foresight, or whether it reflects genuine increases in these cognitions, that is, foreseeability and inevitability hindsight bias.

Hindsight bias as a function of BDI-II and valence. We averaged the three preoutcome foreseeability items and the three pre-outcome inevitability items to composite scores for each participant and each scenario (mean Cronbach's $\alpha=.93$ and .77, respectively). To investigate bias due to outcome knowledge, we subtracted pre-outcome from post-outcome foreseeability and inevitability ratings, respectively. Positive values indicate increased hindsight impressions due to outcome knowledge (i.e., hindsight bias). Results are shown in the lower panels of Figure 1. 
For foreseeability hindsight bias, analyses revealed main effects of BDI-II score, $b=$ $0.65, p<.001$, and outcome valence, $b=13.635, p=.001$. Importantly, there was an interaction, $b=-0.68, p<.001$ : Foreseeability hindsight bias was stronger with higher BDI-II scores after negative outcomes, $b=0.65, p<.001$, but not after positive outcomes, $b=-0.03$, $p=.825$. A similar pattern emerged for inevitability hindsight bias. The main effect of BDI-II score, $b=0.56, p<.001$ was qualified by an interaction of BDI-II score and outcome valence, $b=-0.33, p=.036$ : Inevitability hindsight bias was stronger for higher BDI-II scores after negative outcomes, $b=0.56, p<.001$, but not after positive outcomes, $b=0.23, p=.064$.

It is also worth noting that both analyses yielded negative intercept estimates (foreseeability hindsight bias: $b=-17.21, p<.001$; inevitability hindsight bias: $b=-13.52, p$ $<.001)$. These indicate that for individuals with a BDI-II score of zero, negative outcomes appeared less foreseeable and less inevitable in hindsight than in foresight (i.e., a reverse bias).

Memory hindsight bias. The previous analyses replicated increased impressions of foreseeability and inevitability of negative outcomes in individuals with elevated levels of depression, and additional analyses showed that this "depressive hindsight bias" truly results from receipt of outcome knowledge. Next, we investigated if higher levels of depression were also associated with more pronounced memory hindsight bias.

As a measure of memory hindsight bias, we subtracted participants' initial outcome expectation for each scenario from the corresponding recalled outcome expectation rating after outcome feedback, and scored these differences such that positive values reflected a distortion of the recalled expectation towards the scenario outcome. That is, independent of the valence of the scenario outcome (positive or negative), higher scores indicate memory bias in the direction of the presented outcome. 
Analyses revealed a main effect of BDI-II score, $b=0.19, p=.028$, qualified by an interaction of BDI-II score and valence, $b=-0.34, p=.006$, which was owed to a positive relation of memory bias with BDI-II score for negative outcomes only, $b=0.19, p=.028$ (positive outcomes: $b=-0.14 p=.076$ ).

Hindsight bias: Effects of depression or current mood? The additional mood measure allowed us to investigate if effects of BDI-II score on hindsight cognitions and hindsight bias are attributable to negative mood, a core symptom of depression. For these analyses, we scored the three mood ratings such that higher scores represented more negative mood and averaged them into a composite mood score (Cronbach's $\alpha=.95$ ). Mood expectedly correlated with BDI-II score $(r=.73, p<.001)$.

We first examined if mood had the same effects as BDI-II score on hindsight cognitions and hindsight bias. To this end, we replaced the BDI-II score with the mood score as predictor in the analyses reported above. Replicating interactions of BDI-II score and outcome valence, we found interactions of mood and outcome valence with regard to foreseeability ratings $(b=-0.31 p=.002)$, inevitability ratings $(b=-0.19 p=.009)$, and foreseeability hindsight bias $(b=-0.29 p<.001)$. Contrary to BDI-II, however, mood did not show interactions with outcome valence for inevitability hindsight bias $(b=-0.11 p=.153)$ nor memory hindsight bias $(b=-0.07 p=.267)$.

We then decomposed the significant interactions. In these analyses, we examined whether negative mood alone could explain effects of BDI-II score on hindsight bias. That is, separately for positive and negative outcomes, we compared a model that included mood as the only predictor to a model that included both mood and BDI-II score as predictors. With mood as the only predictor, the pattern of results for the three decomposed interactions were identical to those in the analyses reported above which included BDI-II as the only predictor. When including BDI-II as an additional predictor, the same effects emerged for BDI-II score, 
but, importantly, mood was no longer significant (all $p s>.060$ ). Chi-square likelihood ratio tests showed that including BDI-II in addition to mood improved model fit for negative outcomes (foreseeability ratings: $\Delta \chi^{2}=5.35$, inevitability ratings: $\Delta \chi^{2}=11.33$, foreseeability hindsight bias: $\Delta \chi^{2}=4.07$, all $p \mathrm{~s}<.044$ ), but not for positive outcomes (foreseeability ratings: $\Delta \chi^{2}=0.95$, inevitability ratings: $\Delta \chi^{2}=1.33$, foreseeability hindsight bias: $\Delta \chi^{2}=0.05$, all $p \mathrm{~s}>$ .249). Hence, although negative mood and BDI-II score shared a considerable amount of variance, negative mood alone could not explain the effects of BDI-II score on hindsight bias.

Affect as a function of hindsight bias, responsibility, and BDI-II score. As in Experiment 1, in order to explore affective consequences of hindsight (bias), we analyzed affect ratings (positive affect after positive outcomes, and negative affect after negative outcomes), however, this time as a function of foreseeability hindsight bias, inevitability hindsight bias, and memory hindsight bias (each as predictors in addition to responsibility and BDI-II score).

Negative affect. We analyzed regret as a function of foreseeability hindsight bias, responsibility, and BDI-II score. We found a main effect of responsibility only, $b=0.13, p=$ .015 , indicating greater regret with higher perceived outcome responsibility. A second analysis, focusing on disappointment as a function of inevitability hindsight bias, responsibility, and BDI-II score, yielded a main effect of responsibility, $b=0.14, p=.005$, indicating greater disappointment with higher perceived outcome responsibility, as well as a main effect of BDI-II score, $b=0.49, p=.003$, reflecting greater disappointment with higher levels of depression. Thus, shifts in hindsight cognitions were not associated with negative affect.

Third, we analyzed negative affect (regret and disappointment) as a function of memory hindsight bias, responsibility, and BDI-II score. With respect to regret, only a main effect of responsibility emerged, $b=0.12, p=.025$. A corresponding analysis of 
disappointment yielded main effects of responsibility, $b=0.13, p=.012$, and BDI-II score, $b$ $=0.38, p=.018$. Interestingly, we also found an interaction of memory hindsight bias and BDI-II score, $b=0.01, p=.035$. Decomposition of the interaction revealed that for those with no or low depressive symptoms (BDI-II $\leq 13)$, there was a main effect of responsibility only, $b$ $=0.15, p=.026$. For those with elevated depressive symptoms (BDI-II $>13)$, by contrast, we found - apart from the same main effect of responsibility, $b=0.16, p=.023$ - a main effect of memory hindsight bias, $b=0.31, p=.003$, indicating that stronger memory hindsight bias for negative outcomes was associated with greater disappointment.

Positive affect. We analyzed pride in response to positive scenario outcomes as a function of foreseeability hindsight bias, responsibility, and BDI-II score. Pride depended on responsibility, $b=0.26, p=.007$, and BDI-II score, $b=-0.39, p=.008$; that is, pride was greater with higher perceived outcome responsibility and lower with higher levels of depression. A corresponding analysis of joy as a function of inevitability hindsight bias, responsibility, and BDI-II score yielded a main effect of BDI-II score only, $b=-0.53, p<$ .001 ; that is, there was less joy with higher levels of depression. Thus, just like in Experiment 1, hindsight cognitions (or shift in cognitions) were not associated with positive affect.

We next analyzed positive affect (pride and joy) as a function of memory hindsight bias, responsibility, and BDI-II score. The pride analysis yielded main effects of responsibility, $b=0.23, p=.004$, and BDI-II score, $b=-0.40, p=.005$. That is, as in Experiment 1, pride was greater with higher perceived outcome responsibility and lower with higher levels of depression. Finally, a corresponding analysis of joy showed a main effect of BDI-II score only, $b=-0.55, p<.001$; specifically, joy was greater with lower levels of depression.

In sum, level of depression and perceived responsibility were strongly associated with affective reactions following both positive and negative outcomes. In addition, for negative 
outcomes only, memory hindsight bias was associated with disappointment in those with depressive symptoms.

\section{Relationship of hindsight bias with measures of depressive cognition. The}

previous analyses showed that the three types of hindsight bias for negative outcomes foreseeability, inevitability, and memory hindsight bias - were all increased in participants with elevated levels of depressive symptoms. To relate hindsight bias with measures of depressive cognition, we averaged each of the hindsight bias scores (foreseeability, inevitability, and memory hindsight bias) across the negative outcomes and across the positive outcomes. For the CEQ, CTI, and DAS measures, we calculated sum scores for each participant with complete data ( $n=71,67$, and 72, respectively). Correlations are displayed in Table 3.

All three types of hindsight bias after negative outcomes significantly correlated with all three measures of depressive cognition. For hindsight bias after positive outcomes, the pattern was mixed: Inevitability hindsight bias was positively correlated with the DAS and CEQ, whereas memory hindsight bias was negatively correlated with these two measures, and foreseeability hindsight bias was unrelated to measures of depressive cognition. Further, foreseeability hindsight bias for positive and negative outcomes was positively related, $p=$ .001 ; the same held true for inevitability hindsight bias, $p<.001$. For memory hindsight bias, there was no such relationship, $p=.497$.

\section{Discussion}

Experiment 2 replicated the finding of Experiment 1 that with higher levels of depression negative outcomes - but not positive outcomes - appear more foreseeable and more inevitable in hindsight. We extended this finding by showing that these higher postoutcome impressions (e.g., "I knew all along how the situation would turn out.") represent true shifts in hindsight impressions compared to the pre-outcome impressions (e.g., "I know 
how the situation will turn out."). Individuals with elevated levels of depressive symptoms are thus more prone to hindsight bias for negative outcomes (“depressive hindsight bias”).

By contrast, participants without depressive symptoms showed reverse pre-to-postoutcome shifts for negative outcomes; that is, negative outcomes appeared less foreseeable and inevitable in hindsight compared to foresight. Highly surprising outcomes can diminish or reverse hindsight bias (e.g., Calvillo \& Gomes, 2011), and the result thus presumably reflects healthy cognition in healthy individuals: Negative outcomes violate their generally positive expectations.

Experiment 2 additionally showed that, as level of depression increased, expectations about the scenario outcomes became more negative. This result is not surprising as it reflects the negative view of the future that is typical of depression (cognitive triad; Beck et al., 1979). However, in combination with the result of larger pre-to-post outcome shifts in hindsight impressions, this result is more interesting: When the scenarios indeed turned out negatively (i.e., when negative expectations were met), these outcomes appeared even more foreseeable and more inevitable than they had appeared in foresight. Thus, individuals with depressive symptoms have a negatively biased foresight perspective, and - in addition - an even more negatively biased hindsight perspective.

A novel focus in Experiment 2 was on memory hindsight bias. We measured how much recalled outcome-valence expectations shifted towards the actual outcome, compared to initial expectations. We found this bias to be larger with higher levels of depression, yet again only for negative and not for positive scenario outcomes. The task of recalling a prior estimate without bias requires the inhibition of outcome information, which is factually taskirrelevant. Our result is thus compatible with findings showing that individuals with depressive symptoms have difficulties in suppressing irrelevant information in working memory (Cottencin et al., 2008; Hertel \& Gerstle, 2003), and that these difficulties are 
specifically pronounced for negative content (e.g., Joormann \& Gotlib, 2010). Furthermore, if memory hindsight bias is a by-product of knowledge updating, our results would suggest that knowledge is updated more easily with negative information (i.e., information that is consistent with existing negative schemata).

The result of larger memory hindsight bias for negative outcomes with higher levels of depression is also consistent with the increased inevitability and foreseeability hindsight biases for these outcomes because the bias in recalled expectations may go along with higher subjective probability and foreseeability of the outcome in hindsight. These ideas are supported by our correlational analyses.

Additionally, we showed that our core finding of increased hindsight bias for negative outcomes was in part, but not fully, attributable to negative mood. Specifically, mood was not related to inevitability hindsight bias and memory hindsight bias. The exact mechanisms that underlie increased hindsight bias in depression should thus be subject to further research.

In terms of affective implications of hindsight bias, foreseeability and inevitability bias were not associated with ratings of negative or positive affect. We did, however, find memory hindsight bias to be related to disappointment: For individuals with depressive symptoms, memory hindsight bias for negative outcomes (i.e., recalling an even more negative expectation in hindsight than expressed in foresight) was associated with greater disappointment (whereas there was no such effect on regret). This is remarkable because lowering expectations - prior to outcomes, or retrospectively - is considered an adaptive regulation strategy in terms of protection from disappointment (van Dijk, Zeelenberg, \& van der Pligt, 2003; see also Mellers, 2000, for the role of emotions in the context of decision making). Yet, we found the opposite effect here in individuals with depressive symptoms, suggesting memory hindsight bias to be dysfunctional in these individuals. It is important to note, however, that with the present research design we cannot be sure about the direction of 
a possible causal relationship between hindsight bias and affect. Alternatively, negative affect (here: disappointment) may lead to larger memory hindsight bias for negative outcomes (related to findings on mood-congruent recall, Mathews \& MacLeod, 2005; Rusting \& DeHart, 2000). We will return to the issue of causality in the General Discussion.

Finally, Experiment 2 probed the relationship of hindsight bias with other measures of depressive cognition, and found all aspects - schemata, attitudes, and cognitive errors - to be significantly correlated with all three types of hindsight bias for negative outcomes, whereas measures of hindsight bias for positive outcomes showed a more complex pattern (a positive relation to inevitability hindsight bias, no relation to foreseeability hindsight bias, and a negative relation to memory hindsight bias). Of particular interest, the fact that only inevitability hindsight bias showed a positive relation to depressive cognition for both negative and positive outcomes suggests a more general (i.e., outcome-unspecific) role of inevitability-related cognitions (helplessness, lack of control) in depression (Alloy \& Abramson, 1982; Andersen, 1990).

In sum, the findings of Experiment 2 extend the findings of Experiment 1 and provide additional evidence that hindsight bias - whether measured as shifts in foreseeability and inevitability impressions or as memory distortions - is a core component of depressive cognition.

\section{General Discussion}

Hindsight bias is a robust phenomenon with a long research history. Receipt of outcome information biases the reconstruction of our prior judgments towards the newly gained information - a by-product of updating our knowledge about the world (Hoffrage et al., 2000). In the case of self-relevant, negative outcomes (e.g., a break-up, a layoff), our hindsight view may affect our emotional well-being. Yet, there was virtually no empirical research on the role of hindsight bias in depression. With the present study, we contribute 
new findings to this emerging research area at the intersection of cognitive and clinical science.

Participants in both experiments varied in their level of depressive symptoms, from no or minimal to severe symptoms. They listened to everyday scenarios that ended either positively or negatively. They provided a series of ratings before (Experiment 2) and after each scenario outcome (both experiments), including outcome expectations, impressions of foreseeability and inevitability, and affect.

\section{Cognitive Patterns, Judgment Bias, and Memory Hindsight Bias in Depression}

We identified four depression-specific cognitive patterns, two of which are wellknown from the literature: First, with higher levels of depressive symptoms, individuals took more responsibility for negative, but less responsibility for positive outcomes (Sweeney et al., 1986). Second, with higher levels of depressive symptoms, expectations about event outcomes were more negative; that is, depression was associated with a biased foresight (i.e., future) perspective (Beck et al., 1979). Third, we found two manifestations of hindsight retrospective impressions of foreseeability ("I knew all along how it would turn out") and retrospective impressions of inevitability ("It was virtually determined") - to be more pronounced with higher levels of depressive symptoms. This was the case, however, for negative outcomes only. Prior research on healthy individuals, by comparison, found decreased foreseeability (defensive processing) and increased inevitability (retroactive pessimism) after negative, self-relevant outcomes. The present research extends and qualifies these findings by showing that higher levels of depression go along with a detrimental combination of both increased foreseeability and increased inevitability of negative outcomes. Viewing a past, negative event as foreseeable suggests the possibility of personal influence or control - thereby inviting regret or self-blame, but also the possibility of improvement of actions and decisions in the future. Viewing the event outcome as inevitable 
at the same time, however, precludes personal influence and thus future improvement, and therefore most probably invites feelings of helplessness. Results also showed that this view resulted from the receipt of outcome knowledge, that is, true hindsight bias. Thus, depressive symptoms are associated with negative future expectations, and - in case these are met (i.e., when outcomes are negative) - additionally with distorted hindsight impressions of foreseeability and inevitability (“depressive hindsight bias”).

Finally, a fourth depression-specific distortion emerged in the form of increased memory hindsight bias for earlier judgments of expectations. Participants with higher levels of depression recalled their already negative expectations about future outcomes as even more negative after knowing that the outcome was indeed negative. Importantly, this bias was also absent for positive outcomes.

\section{Implications}

Negatively biased responsibility attributions and future expectations concur with cognitive theories of depression (Clark, Beck, \& Alford, 1999) and theories of helplessness in depression (Abramson et al., 1978; Alloy \& Abramson, 1982). Beyond this, our finding of a "depressive hindsight bias" underscores that negative thoughts of depressed individuals do not only center on the world, the self, and the future ("cognitive triad", Beck \& Bredemeier, 2016; Beck et al., 1979), but also on the past. This retrospectively amplified negative view of the past - in terms of exaggerated foreseeability and inevitability of negative outcomes, as well as pessimistically distorted memories of initial expectations - does not necessarily follow from negative schemata of the world, the self, or the future. Specifically, it is conceivable that individuals support their negative views of the future, the (current) self, or the state of the world by contrasting them with a (remembered-as-)better past ("Things were better in the old days.”, e.g., Mitchell, Thompson, Peterson, \& Cronk, 1997). This is, however, not what we found for individuals with depression; they held consistently negative 
hindsight views. Therefore, we propose a negative view of the past, including depressive hindsight bias, as a manifestation of depressive cognition in its own right, complementing and extending the other three biases into a "cognitive tetrad".

Although this negative view of the past is conceptually distinct from the other three schemata, all four are expected to be interrelated in depression, insofar as they are all part of the depressive syndrome. This idea is supported by the correlations we found between hindsight bias and other, established measures of depressive cognition (notably the Cognitive Triad Inventory, but also the DAS and the CEQ).

Hindsight bias as a cognitive distortion thus plays a major role in depressive thinking, because it applies to judgments of past events (inevitability), metacognitive judgments (foreseeability), the way these judgments change with outcome knowledge (judgment shifts), and the way judgments are recalled upon receipt of outcome knowledge (memory hindsight bias). Yet, we suggest that depressed individuals' hindsight bias will not only cloud their representation of the past but will also strongly impact their thinking about the future. Specifically, hindsight bias will preserve and amplify depression-related cognitive biases, because each experience of a negative outcome will strengthen the representation of those aspects of the (former) foresight perspective that are in line with the negative outcome, whereas aspects that are in support of alternative positive outcomes will be further weakened. Thus, just as hindsight bias may indicate adaptive learning and knowledge-updating in affectpoor situations, it may contribute considerably to the corroboration of negative thought content, that is, to maladaptive learning and knowledge-updating in affect-rich (i.e., negative and self-relevant) situations in individuals with depression.

If evidence for such a mechanism can be established with clinical samples in future studies, it will be very important to address hindsight bias in psychotherapy. The modification of biased cognitive representations of past events is an important element of 
cognitive-behavioral interventions for any client suffering from depression or trauma, but is even more important when an individual's future time perspective is limited (e.g., with old age or terminal illness). In such situations, a negatively biased view of one's biography puts particular strain on the person. Life-review approaches (Butler, 1963; Serrano, Latorre, Gatz, \& Montanes, 2004) explicitly target this biased view of the past and help the individual attain a differentiated and balanced representation of positive and negative life experiences. Our research suggests that depressive hindsight bias - as a core aspect of depressive cognition may be a major obstacle to such reappraisals.

A related question is how hindsight bias is linked to affective responding. We did not find support for a role of foreseeability and inevitability impressions in affect regulation. We did, however, find that in those with elevated depressive symptoms, disappointment after negative outcomes was greater if the expectations held earlier were recalled as more negative. Memory hindsight bias may thus also be a promoter of negative affect, specifically in depression. However, this rather counterintuitive effect awaits replication in further studies.

\section{Limitations}

There are some caveats, however, when trying to draw clear conclusions from our experiments regarding the effect of hindsight on affective reactions. First, the validity of the affect ratings that followed each scenario may be limited. Our scenarios and the corresponding affect ratings were hypothetical in nature. Future research could therefore examine the relationship between hindsight bias and affective reactions in studies with genuine positive or negative experiences (e.g., by giving participants positive or negative feedback in an experimental task, or by investigating real-life events). Moreover, depression is associated with weak affective responding and anhedonia, with difficulties in recognizing and verbalizing affective states (alexithymia; e.g., Honkalampi, Hintikka, Tanskanen, Lehtonen, \& Viinamäki, 2000; Taylor \& Bagby, 2004), and with negatively biased mood 
predictions (affective forecasting; e.g., Wenze, Gunthert, \& German, 2012). That is, depression may additionally limit the validity of affect ratings.

Second, the causal direction of the link between hindsight bias and affective responding remains ambiguous with our research design. For example, whereas larger memory hindsight bias after negative outcomes may lead to greater disappointment, disappointment may also increase the probability of a negatively biased recall (Mathews \& MacLeod, 2005). Future research could therefore attempt to manipulate the strength of foreseeability and inevitability impressions by manipulating the feeding factors (e.g., surprise at the outcomes, causal connection of the outcome determinants), and measure affective responses before and after the manipulation and measurement of hindsight cognitions.

Third, the present research cannot tell us how realistic the observed foresight expectations and hindsight views are on an absolute level (Alloy \& Abramson, 1988; Strunk, Lopez, \& DeRubeis, 2006). Specifically, it is unclear whether these cognitions are indeed unrealistically biased in a negative direction in depressed individuals, or unrealistically biased in a positive direction in non-depressed individuals (see Moore \& Fresco, 2012, for a metaanalysis on depressive realism).

Finally, the current study is limited with regard to generalizability to populations other than university students. Future studies should therefore investigate whether the results replicate within the general population and with clinical samples.

\section{Outlook}

A question to address in future research is whether hindsight bias is a general processing tendency (i.e., a cognitive trait). The current research investigated hindsight bias regarding negative and self-referential content. In a different study (Groß \& Bayen, 2016), we used a different type of materials and content and showed that participants with increased levels of depressive symptoms exhibited a higher probability of biased reconstructions of 
their prior numerical estimates to (emotionally neutral) general knowledge questions, compared to controls. If hindsight bias is a cognitive trait, there should be a relationship between hindsight bias for different types of material and content. In a related context, Takarangi, Smith, Strange, and Flowe (2016) showed that individuals holding maladaptive metacognitions before trauma exposure had an increased risk of PTSD symptoms after exposure. Similarly, if proneness to hindsight bias (in whatever form) is a stable cognitive trait, it may not only constitute a consequence of depressive symptoms, but also a vulnerability factor for depression. 


\section{Authorship}

Julia Groß developed the study concept. All authors contributed to the study design. The experiments were performed in the laboratory of the Mathematical and Cognitive Psychology group at Heinrich-Heine-Universität Düsseldorf (Director Ute J. Bayen). Data collections were performed by students Anna-Christina Breuer, Doris Dahlke, and Laura Richter, under the supervision of Julia Groß. Julia Groß also performed the data analysis and interpretation; Hartmut Blank and Ute J. Bayen provided additional suggestions for analysis and interpretation. Julia Groß drafted the paper, and Hartmut Blank and Ute J. Bayen provided critical revisions. All authors approved the final version of the paper for submission. 


\section{References}

Abramson, L. Y., Seligman, M. E., \& Teasdale, J. D. (1978). Learned helplessness in humans: Critique and reformulation. Journal of Abnormal Psychology, 87, 49-74. doi: 10.1037//0021-843X.87.1.49

Alba, J. W., \& Hasher, L. (1983). Is memory schematic? Psychological Bulletin, 93, 203231. doi:10.1037/0033-2909.93.2.203

Alicke, M. D. (2000). Culpable control and the psychology of blame. Psychological Bulletin, 126, 556-574. doi:10.1037/0033-2909.126.4.556

Alloy, L. B., \& Abramson, L. Y. (1982). Learned helplessness, depression, and the illusion of control. Journal of Personality and Social Psychology, 42, 1114-1126. doi:10.1037/0022-3514.42.6.1114

Alloy, L. B., \& Abramson, L. Y. (1988). Depressive realism: Four theoretical perspectives. In L. B. Alloy \& L. Y. Abramson (Eds.), Cognitive processes in depression (pp. 223265). New York, NY, US: Guilford Press.

Andersen, S. M. (1990). The inevitability of future suffering: The role of depressive predictive certainty in depression. Social Cognition, 8, 203-228. doi:10.1521/soco.1990.8.2.203

Arkes, H. R. (2013). The consequences of the hindsight bias in medical decision making. Psychological Science, 22, 356-360. doi:10.1177/0963721413489988

Bates, D., Maechler, M., Bolker, B., \& Walker, S. (2015). Fitting linear mixed-effects models uing lme4. Journal of Statistical Software, 67, 1-48. doi:10.18637/jss.v067.i01.

Barr, D. J., Levy, R., Scheepers, C., \& Tily, H. J. (2013). Random effects structure for confirmatory hypothesis testing: Keep it maximal. Journal of Memory and Language, 68, 255-278. doi:10.1016/j.jml.2012.11.001

Bartlett, F. C. (1932). Remembering: A study in experimental and social psychology. 
Cambridge, England: Cambridge University Press.

Bayen, U. J., Nakamura, G. V., Dupuis, S. E., \& Yang, C.-L. (2000). The use of schematic knowledge about sources in source monitoring. Memory \& Cognition, 28, 480-500. doi:10.3758/BF03198562

Beck, A. T., \& Bredemeier, K. (2016). A unified model of depression: Integrating clinical, cognitive, biological, and evolutionary perspectives. Clinical Psychological Science, 4, 596-619. doi:10.1177/2167702616628523

Beck, A. T., Rush, A. J., Shaw, B. F., \& Emery, G. (1979). Cognitive therapy of depression. New York: Guilford.

Beck, A. T., Steer, R. A., \& Brown, G. K. (1996). Manual for the Beck Depression Inventory, 2nd ed. San Antonio, TX: The Psychological Corporation.

Beckham, E. E., Leber, W. R., Watkins, J. T., Boyer, J. L., \& Cook, J. B. (1986).

Development of an instrument to measure Beck's cognitive triad: The Cognitive Triad Inventory. Journal of Consulting and Clinical Psychology, 54, 566-567. doi:10.1037/0022-006X.54.4.566

Blank, H., Fischer, V., \& Erdfelder, E. (2003). Hindsight bias in political elections. Memory, 11, 491-504. doi:10.1080/09658210244000513

Blank, H., \& Nestler, S. (2006). Perceiving events as both inevitable and unforeseeable in hindsight: The Leipzig candidacy for the Olympics. British Journal of Social Psychology, 45, 149-160. doi:10.1348/014466605X52326

Blank, H., Nestler, S., von Collani, G., \& Fischer, V. (2008). How many hindsight biases are there? Cognition, 106, 1408-40. doi:10.1016/j.cognition.2007.07.007

Blank, H., \& Peters, J. H. (2010). Controllability and hindsight components: Understanding opposite hindsight biases for self-relevant negative event outcomes. Memory \& Cognition, 38, 356-65. doi:10.3758/MC.38.3.356 
Brewer, W. F. (2000). Bartlett's concept of the schema and its impact on theories of knowledge representation in contemporary cognitive psychology. In A. Saito (Ed.), Bartlett, culture and cognition (pp. 69-89). New York, NY: Psychology Press.

Butler, R. N. (1963). The life review: An interpretation of reminiscence in the aged. Psychiatry, 26, 65-76. doi:10.1080/00332747.1963.11023339

Calvillo, D. P., \& Gomes, D. M. (2011). Surprise influences hindsight-foresight differences in temporal judgments of animated automobile accidents. Psychonomic Bulletin \& Review, 18, 385-91. doi:10.3758/s13423-011-0062-4

Cantor, N., \& Mischel, W. (1977). Traits as prototypes: Effects on recognition memory. Journal of Personality and Social Psychology, 35, 38-48. doi:10.1037/00223514.35.1.38

Carli, L. L. (1999). Cognitive reconstruction, hindsight, and reactions to victims and perpetrators. Personality and Social Psychology Bulletin, 25, 966-979. doi:10.1177/01461672992511005

Clark, D. A., Beck, A. T., \& Alford, B. A. (1999). Scientific foundations of cognitive theory and therapy of depression. New York, NY: Wiley.

Cottencin, O., Gruat, G., Thomas, P., Devos, P., Goudemand, M., \& Consoli, S. M. (2008). Directed forgetting in depression. Journal of the International Neuropsychological Society, 14, 895-899. doi:10.1017/S1355617708081186

Enders, C. K., \& Tofighi, D. (2007). Centering predictor variables in cross-sectional multilevel models: A new look at an old issue. Psychological Methods, 12, 121-138. doi:10.1037/1082-989X.12.2.121

Erdfelder, E., \& Buchner, A. (1998). Decomposing the hindsight bias: A multinomial processing tree model for separating recollection and reconstruction in hindsight. Journal of Experimental Psychology: Learning, Memory, and Cognition, 24, 387-414. 
doi:10.1037//0278-7393.24.2.387

Gotlib, I. H., \& Joormann, J. (2010). Cognition and depression: Current status and future directions. Annual Review of Clinical Psychology, 6, 285-312. doi:10.1146/annurev.clinpsy.121208.131305.Cognition

Groß, J., \& Bayen, U. J. (2016). Effects of dysphoria and induced negative mood on the processes underlying hindsight bias. Cognition and Emotion. Advance online publication. doi:10.1080/02699931.2016.1249461

Hankin, B. L., Fraley, R. C., Lahey, B. B., \& Waldman, I. D. (2005). Is depression best viewed as a continuum or discrete category? A taxometric analysis of childhood and adolescent depression in a population-based sample. Journal of Abnormal Psychology, 114, 96-110. doi:10.1037/0021-843X.114.1.96

Haslam, N., \& Jayasinghe, N. (1995). Negative affect and hindsight bias. Journal of Behavioral Decision Making, 8, 127-135. doi:10.1002/bdm.3960080205

Hautzinger, M., Keller, F., \& Kühner, C. (2006). Das Beck Depressionsinventar II. Frankfurt am Main: Harcourt Test Services.

Hawkins, S. A., \& Hastie, R. (1990). Hindsight: Biased judgments of past events after the outcomes are known. Psychological Bulletin, 107, 311-327. doi:10.1037//00332909.107.3.311

Hertel, P. T., \& Gerstle, M. (2003). Depressive deficits in forgetting. Psychological Science, 14, 573-578. doi:10.1046/j.0956-7976.2003.psci_1467.x

Hoffman, L., \& Rovine, M. J. (2007). Multilevel models for the experimental psychologist: Foundations and illustrative examples. Behavior Research Methods, 39, 101-117. doi: 10.3758/BF03192848

Hoffrage, U., Hertwig, R., \& Gigerenzer, G. (2000). Hindsight bias: A by-product of knowledge updating? Journal of Experimental Psychology. Learning, Memory, and 
Cognition, 26, 566-81. doi: 10.1093/acprof:oso/9780199744282.003.0010

Honkalampi, K., Hintikka, J., Tanskanen, A., Lehtonen, J., \& Viinamäki, H. (2000).

Depression is strongly associated with alexithymia in the general population. Journal of Psychosomatic Research, 48, 99-104. doi:10.1016/S0022-3999(99)00083-5

Joormann, J., \& Gotlib, I. H. (2010). Emotion regulation in depression: Relation to cognitive inhibition. Cognition and Emotion, 24, 281-298. doi:10.1080/02699930903407948

Kubany, E. S., \& Manke, F. P. (1995). Cognitive therapy for trauma-related guilt: Conceptual bases and treatment outlines. Cognitive and Behavioral Practice, 2, 27-61. doi:10.1016/S1077-7229(05)80004-5

Kuhlmann, B.G., Vaterrodt, B., \& Bayen, U. J. (2012). Schema bias in source monitoring varies with encoding conditions: Support for a probability-matching account. Journal of Experimental Psychology: Learning, Memory, and Cognition, 28, 1365-1376. doi:10.1037/a0028147

Kuznetsova, A., Brockhoff, P. B., \& Christensen, R. H. B. (2015). lmerTest: Tests in Linear Mixed Effects Models. R Package. Retrieved from http://cran.rproject.org/package $=$ lmerTest

Leahy, R. L. (2004). Decision making and psychopathology. In R. L. Leahy (Ed.), Contemporary cognitive therapy: Theory, research, and practice (pp. 116-140). New York, NY: Guilford Press.

Lefebvre, M. F. (1981). Cognitive distortion and cognitive errors in depressed psychiatric and low back pain patients. Journal of Consulting and Clinical Psychology, 49, 517-525. doi:10.1037/0022-006X.49.4.517

Louie, T. A. (1999). Decision makers' hindsight bias after receiving favorable and unfavorable feedback. Journal of Applied Psychology, 84, 29-41. doi:10.1037/00219010.84.1.29 
Louie, T. A. (2005). Hindsight bias and outcome-consistent thoughts when observing and making service provider decisions. Organizational Behavior and Human Decision Processes, 98, 88-95. doi:10.1016/j.obhdp.2005.03.004

Louie, T. A., Curren, M. T., \& Harich, K. R. (2000). “I knew we would win”: Hindsight bias for favorable and unfavorable team decision outcomes. Journal of Applied Psychology, 85, 264-272. doi:10.1037//0021-9010.85.2.264

MacCallum, R. C., Zhang, S., Preacher, K. J., \& Rucker, D. D. (2002). On the practice of dichotomization of quantitative variables. Psychological Methods, 7, 19-40. doi:10.1037//1082-989X.7.1.19

Mark, M. M., Boburka, R. R., Eyssell, K. M., Cohen, L. L., \& Mellor, S. (2003). “I couldn’t have seen it coming": The impact of negative self-relevant outcomes on retrospections about foreseeability. Memory, 11, 443-454. doi:10.1080/09658210244000522

Mark, M. M., \& Mellor, S. (1991). Effect of self-relevance of an event on hindsight bias: The foreseeability of a layoff. Journal of Applied Psychology, 76, 569-577. doi:10.1037//0021-9010.76.4.569

Mathews, A., \& MacLeod, C. (2005). Cognitive vulnerability to emotional disorders. Annual Review of Clinical Psychology, 1, 167-95. doi:10.1146/annurev.clinpsy.1.102803.143916

Mellers, B. (2000). Choice and the relative pleasure of consequences. Psychological Bulletin, 126, 910-924. doi:10.1037//0033-2909.126.6.910

Mitchell, T. R., Thompson, L., Peterson, E., \& Cronk, R. (1997). Temporal adjustments in the evaluation of events: The "rosy view". Journal of Experimental Social Psychology, 33, 421-448. doi:10.1006/jesp.1997.1333

Moore, M. T., \& Fresco, D. M. (2012). Depressive realism: A meta-analytic review. Clinical Psychology Review, 32, 496-509. doi:10.1016/j.cpr.2012.05.004 
Musch, J., \& Wagner, T. (2007). Did everybody know it all along? A review of individual differences in hindsight bias. Social Cognition, 25, 64-82. doi: $10.1521 /$ soco.2007.25.1.64

Nestler, S., Blank, H., \& Egloff, B. (2010). Hindsight $\neq$ hindsight: Experimentally induced dissociations between hindsight components. Journal of Experimental Psychology. Learning, Memory, and Cognition, 36, 1399-413. doi:10.1037/a0020449

Oeberst, A., von der Beck, I., \& Nestler, S. (2014). Reading about explanations enhances perceptions of inevitability and foreseeability: A cross-cultural study with Wikipedia articles. Cognitive Processing, 15, 343-349. doi:10.1007/s10339-014-0603-7

Pezzo, M. V, \& Beckstead, J. W. (2008). The effects of disappointment on hindsight bias for real-world outcomes. Applied Cognitive Psychology, 22, 491-506. doi: 10.1002/acp. 1377

Pohl, R. F., Eisenhauer, M., \& Hardt, O. (2003). SARA: A cognitive process model to simulate the anchoring effect and hindsight bias. Memory, 11, 337-56. doi:10.1080/09658210244000487

Pössel, P. (2008). Cognitive Error Questionnaire (CEQ): Psychometric properties and factor structure of the German translation. Journal of Psychopathology and Behavioral Assessment, 31, 264-269. doi:10.1007/s10862-008-9117-x

Pössel, P. (2009). Cognitive Triad Inventory (CTI): Psychometric properties and factor structure of the German translation. Journal of Behavior Therapy and Experimental Psychiatry, 40, 240-247. doi:10.1016/j.jbtep.2008.12.001

Power, M. J., Katz, R., McGuffin, P., Duggan, C. F., Lam, D., \& Beck, A. T. (1994). The Dysfunctional Attitude Scale (DAS): A comparison of forms A and B and proposals for a new subscaled version. Journal of Research in Personality, 28, 263-276. doi:10.1006/jrpe.1994.1019 
Renner, B. (2003). Hindsight bias after receiving self-relevant health risk information: A motivational perspective. Memory, 11, 455-72. doi:10.1080/09658210244000531

Roese, N. J., \& Maniar, S. D. (1997). Perceptions of purple: Counterfactual and hindsight judgments at Northwestern Wildcats football games. Personality and Social Psychology Bulletin, 23, 1245-1253. doi: 10.1177/01461672972312002

Roese, N. J., \& Vohs, K. D. (2012). Hindsight Bias. Perspectives on Psychological Science, 7, 411-426. doi:10.1177/1745691612454303

Rojas, R., Geissner, E., \& Hautzinger, M. (2015). DAS-18 Form A und Form B. Diagnostica, 61, 173-183. doi:10.1026/0012-1924/a000123

Ruscio, J., \& Ruscio, A. M. (2000). Informing the continuity controversy: A taxometric analysis of depression. Journal of Abnormal Psychology, 109, 473-487. doi: 10.1037//0021-843X.109.3.473

Rusting, C. L., \& DeHart, T. (2000). Retrieving positive memories to regulate negative mood: Consequences for mood-congruent memory. Journal of Personality and Social Psychology, 78, 737-752. doi:10.1037//0022-3514.78

Serrano, J. P., Latorre, J. M., Gatz, M., \& Montanes, J. (2004). Life review therapy using autobiographical retrieval practice for older adults with depressive symptomatology. Psychology and Aging, 19, 270-277. doi:10.1037/0882-7974.19.2.272

Slovic, P., \& Fischhoff, B. (1977). On the psychology of experimental surprises. Journal of Experimental Psychology: Human Perception and Performance, 3, 544-551. doi:10.1037//0096-1523.3.4.544

Strunk, D. R., Lopez, H., \& DeRubeis, R. J. (2006). Depressive symptoms are associated with unrealistic negative predictions of future life events. Behaviour Research and Therapy, 44, 861-82. doi:10.1016/j.brat.2005.07.001

Sweeney, P. D., Anderson, K., \& Bailey, S. (1986). Attributional style in depression: A meta- 
analytic review. Journal of Personality and Social Psychology, 50, 974-991. doi: $10.1037 / / 0022-3514.50 .5 .974$

Takarangi, M. K. T., Smith, R. A., Strange, D., \& Flowe, H. D. (2016). Metacognitive and metamemory beliefs in the development and maintenance of posttraumatic stress disorder. Clinical Psychological Science. Advance online publication. doi: $10.1177 / 2167702616649348$

Taylor, G. J., \& Bagby, R. M. (2004). New trends in alexithymia research. Psychotherapy and Psychosomatics, 73, 68-77. doi:10.1159/000075537

Tykocinski, O. E. (2001). I never had a chance: Using hindsight tactics to mitigate disappointments. Personality and Social Psychology Bulletin, 27, 376-382. doi:10.1177/0146167201273011

Tykocinski, O. E., Pick, D., \& Kedmi, D. (2002). Retroactive pessimism: A different kind of hindsight bias. European Journal of Social Psychology, 32, 577-588. doi:10.1002/ejsp.120

Tykocinski, O. E., \& Steinberg, N. (2005). Coping with disappointing outcomes: Retroactive pessimism and motivated inhibition of counterfactuals. Journal of Experimental Social Psychology, 41, 551-558. doi:10.1016/j.jesp.2004.12.001

Üstün, T. B., Ayuso-Mateos, J. L., Chatterji, S., Mathers, C., \& Murray, C. J. L. (2014). Global burden of depressive disorders in the year 2000. The British Journal of Psychiatry, 184, 386-392. doi:10.1192/bjp.184.5.386

van Dijk, W. W., Zeelenberg, M., \& van der Pligt, J. (2003). Blessed are those who expect nothing: Lowering expectations as a way of avoiding disappointment. Journal of Economic Psychology, 24, 505-516. doi:10.1016/S0167-4870(02)00211-8

Wann, D. L., Grieve, F. G., Waddill, P. J., \& Martin, J. (2008). Use of retroactive pessimism as a method of coping with identity threat: The impact of group identification. Group 
Processes \& Intergroup Relations, 11, 439-450. doi:10.1177/1368430208095399

Wenze, S. J., Gunthert, K. C., \& German, R. E. (2012). Biases in affective forecasting and recall in individuals with depression and anxiety symptoms. Personality and Social Psychology Bulletin, 38, 895-906. doi:10.1177/0146167212447242

Williams, J. M. G. (1992). The psychological treatment of depression (2nd ed). London: Routledge.

Wittchen, H.-U., \& Jacobi, F. (2005). Size and burden of mental disorders in Europe - A critical review and appraisal of 27 studies. European Neuropsychopharmacology, 15, 357-376. doi:10.1016/j.euroneuro.2005.04.012

Zeelenberg, M., van Dijk, W. W., \& Manstead, A. S. R. (1998). Reconsidering the relation between regret and responsibility. Organizational Behavior and Human Decision Processes, 74, 254-272. doi:10.1006/obhd.1998.2780

Zeelenberg, M., Van Dijk, W. W., Manstead, A. S. R., \& van der Pligt, J. (2000). On bad decisions and disconfirmed expectancies: The psychology of regret and disappointment. Cognition and Emotion, 14, 521-541. doi:10.1080/026999300402781 


\section{Footnotes}

${ }^{1}$ We faced convergence problems of the maximum likelihood estimation algorithm in some of the analyses that involved two within-participant/within-scenario predictors. In these cases, we followed the suggestions by Barr, Levy, Scheepers, and Tily (2013) and simplified the maximal random-effects structure such that we first removed correlations between random intercepts and random slopes, and next removed those random slopes that had the smallest random-effects variance according to the statistics of the partially converged model.

${ }^{2}$ We performed MLM analyses in R using the lmer() function from the lme4 package (Bates, Maechler, Bolker, \& Walker, 2015) and obtained $p$ values using the lmerTest package (Kuznetsova, Brockhoff, \& Christensen, 2015), which uses Satterthwaite approximations to degrees of freedom.

${ }^{3}$ A reviewer suggested the possibility that differences in imaginability might (partially) explain effects of depressive symptoms on hindsight cognitions and/or hindsight bias. We therefore performed additional analyses of outcome expectation, hindsight cognitions, and hindsight bias, with imaginability as a control predictor. The pattern of results remained the same. 
Table 1

Characteristics of Participants in Experiments 1 and 2

\begin{tabular}{|c|c|c|c|c|c|c|c|c|c|}
\hline & \multirow[t]{2}{*}{$N$} & \multirow{2}{*}{$\begin{array}{c}\text { Gender } \\
\text { Female }\end{array}$} & \multicolumn{2}{|c|}{ Age } & \multicolumn{5}{|c|}{ BDI-II } \\
\hline & & & $M$ & $S D$ & $M$ & Range & $14-19$ & $20-28$ & $>28$ \\
\hline \multicolumn{10}{|l|}{ Experiment 1} \\
\hline BDI-II $\leq 13$ & 34 & 25 & 22.0 & 4.1 & 4.3 & $0-13$ & & & \\
\hline BDI-II >13 & 26 & 21 & 22.4 & 3.6 & 19.9 & $14-38$ & $62 \%$ & $30 \%$ & $8 \%$ \\
\hline \multicolumn{10}{|l|}{ Experiment 2} \\
\hline BDI-II $\leq 13$ & 45 & 38 & 20.9 & 3.0 & 5.4 & $0-13$ & & & \\
\hline BDI-II $>13$ & 30 & 24 & 22.3 & 3.7 & 23.1 & $15-38$ & $40 \%$ & $37 \%$ & $23 \%$ \\
\hline
\end{tabular}

Note. BDI-II = Beck Depression Inventory, 2nd edition. According to Beck et al. (1996), a score of

14-19 indicates mild, 20-28 moderate, and > 28 severe depressive symptoms. 
Table 2

Descriptive Statistics

\begin{tabular}{|c|c|c|c|c|c|c|c|c|c|c|}
\hline \multirow[b]{4}{*}{$\mathrm{BDI}-\mathrm{II} \leq 13$} & \multirow{2}{*}{\multicolumn{4}{|c|}{$\begin{array}{l}\text { Experiment } 1 \\
\text { post-outcome }\end{array}$}} & \multicolumn{6}{|c|}{ Experiment 2} \\
\hline & & & & & \multicolumn{2}{|c|}{ pre-outcome } & \multicolumn{4}{|c|}{ post-outcome } \\
\hline & \multicolumn{2}{|c|}{ positive } & \multicolumn{2}{|c|}{ negative } & \multirow[b]{2}{*}{$M$} & \multirow[b]{2}{*}{$S D$} & \multicolumn{2}{|c|}{ positive } & \multicolumn{2}{|c|}{ negative } \\
\hline & $M$ & $S D$ & $M$ & $S D$ & & & $M$ & $S D$ & $M$ & $S D$ \\
\hline Foreseeability $^{\mathrm{a}}$ & 41.2 & 14.8 & 37.0 & 13.8 & 58.0 & 28.3 & 56.1 & 17.3 & 44.5 & 12.5 \\
\hline Inevitability $^{\mathrm{a}}$ & 34.6 & 13.8 & 28.5 & 12.9 & 43.4 & 28.1 & 37.2 & 15.2 & 30.7 & 22.9 \\
\hline Responsibility & 60.0 & 16.9 & 50.5 & 15.8 & \multicolumn{2}{|c|}{-} & 61.5 & 11.9 & 50.9 & 13.9 \\
\hline Pride / Regret & 68.3 & 14.0 & 75.6 & 12.9 & \multicolumn{2}{|c|}{-} & 67.8 & 13.0 & 72.6 & 18.9 \\
\hline Joy / Disapp. & 94.3 & 6.2 & 77.5 & 13.4 & \multicolumn{2}{|c|}{-} & 92.5 & 6.6 & 74.7 & 16.7 \\
\hline $\begin{array}{l}\text { (Recalled) } \\
\text { expectation }^{b}\end{array}$ & \multicolumn{2}{|c|}{-} & \multicolumn{2}{|c|}{-} & 68.9 & 26.0 & 68.9 & 28.3 & 66.2 & 26.7 \\
\hline \multicolumn{11}{|l|}{ BDI-II> 13} \\
\hline Foreseeability $^{\mathrm{a}}$ & 39.2 & 9.3 & 49.4 & 17.5 & 54.2 & 28.8 & 46.7 & 16.2 & 52.0 & 19.2 \\
\hline Inevitability $^{\mathrm{a}}$ & 33.0 & 9.6 & 42.9 & 17.2 & 44.3 & 29.1 & 39.6 & 17.2 & 44.5 & 17.3 \\
\hline Responsibility & 52.8 & 14.6 & 65.8 & 13.8 & \multicolumn{2}{|c|}{-} & 51.5 & 19.8 & 65.0 & 14.6 \\
\hline Pride / Regret & 62.1 & 14.0 & 77.3 & 13.4 & \multicolumn{2}{|c|}{-} & 57.8 & 21.7 & 75.0 & 18.8 \\
\hline Joy / Disapp. & 83.7 & 12.8 & 80.2 & 12.2 & & & 81.5 & 16.4 & 83.6 & 11.1 \\
\hline $\begin{array}{l}\text { (Recalled) } \\
\text { expectation }^{\mathrm{b}}\end{array}$ & \multicolumn{2}{|c|}{-} & \multicolumn{2}{|c|}{-} & 53.2 & 27.8 & 51.4 & 27.8 & 47.1 & 30.0 \\
\hline
\end{tabular}


Note. BDI-II = Beck Depression Inventory, second edition. All ratings were assessed on a scale from 0 to 100 , with higher ratings indicating higher levels of the measured variable. For cells filled with a dash ('-'), no data were collected.

${ }^{a}$ Presented are means for the foreseeability and inevitability items, respectively. ${ }^{\mathrm{b}}$ The presented values indicate pre-outcome expectations and recalled post-outcome expectations. Higher values indicate a more positive (recalled) expectation. 
Table 3

Correlations between measures of depressive cognition and hindsight bias in Experiment 2

\begin{tabular}{|c|c|c|c|c|c|c|c|c|c|c|c|}
\hline & Measure & 1 & 2 & 3 & 4 & 5 & 6 & 7 & 8 & 9 & 10 \\
\hline 1 & BDI-II & - & $.87 *$ & $.73^{*}$ & $.79 *$ & $.45^{*}$ & $.41 *$ & $.25 *$ & -.01 & .20 & -.20 \\
\hline 2 & CTI & & - & $.81 *$ & $.78^{*}$ & $.53 *$ & $.52 *$ & $.40^{*}$ & -.04 & .22 & -.18 \\
\hline 3 & DAS & & & - & $.68^{*}$ & $.45^{*}$ & $.36^{*}$ & $.27 *$ & -.01 & $.25^{*}$ & $-.26^{*}$ \\
\hline 4 & CEQ & & & & - & $.54 *$ & $.50^{*}$ & $.31 *$ & .13 & $.23^{*}$ & $-.38 *$ \\
\hline 5 & Foreseeability HB (neg) & & & & & - & $.63^{*}$ & $.25^{*}$ & $.36^{*}$ & $.29 *$ & -.18 \\
\hline 6 & Inevitability HB (neg) & & & & & & - & $.27 *$ & $.25^{*}$ & $.38 *$ & $-.29 *$ \\
\hline 7 & Memory HB (neg) & & & & & & & - & .11 & .06 & -.08 \\
\hline 8 & Foreseeability HB (pos) & & & & & & & & - & $.50^{*}$ & .11 \\
\hline 9 & Inevitability HB (pos) & & & & & & & & & - & -.15 \\
\hline 10 & Memory HB (pos) & & & & & & & & & & - \\
\hline
\end{tabular}

Note. BDI-II = Beck Depression Inventory, second edition; CTI = Cognitive Triad Inventory; DAS = Dysfunctional Attitudes Scale; CEQ =

Cognitive Errors Questionnaire; HB = Hindsight Bias; neg/pos = negative/positive outcomes.

$* p<.05$ 
Figure Captions

Figure 1. Foreseeability (left) and inevitability (right) ratings (Experiment 1, post-outcome) and foreseeability and inevitability hindsight bias (Experiment 2, post-outcome rating minus pre-outcome rating) as a function of BDI-II score and valence of the scenario outcome.

Individual dots represent scenario averages for each participant. $* p<.05$. 